\title{
Consequences of being overweight or obese during pregnancy on diabetes in the offspring: a record linkage study in Aberdeen, Scotland
}

\author{
Marius Lahti-Pulkkinen ${ }^{1,2,3} \cdot$ Sohinee Bhattacharya ${ }^{4} \cdot$ Sarah H. Wild $^{5} \cdot$ Robert S. Lindsay $^{6} \cdot$ Katri Räikkönen $^{3}$. \\ Jane E. Norman ${ }^{2}$ • Siladitya Bhattacharya ${ }^{4}$ • Rebecca M. Reynolds ${ }^{1,2}$
}

Received: 20 September 2018 / Accepted: 20 March 2019/Published online: 19 June 2019

(C) The Author(s) 2019

\begin{abstract}
Aims/hypothesis Maternal obesity in pregnancy is associated with cardiovascular disease and mortality rate in the offspring. We aimed to determine whether maternal obesity is also associated with increased incidence of type 2 and type 1 diabetes in the offspring, independently of maternal diabetes as a candidate mechanistic pathway.

Methods Birth records of 118,201 children from 1950 to 2011 in the Aberdeen Maternity and Neonatal Databank were linked to Scottish Care Information-Diabetes, the national register for diagnosed diabetes in Scotland, to identify incident and prevalent type 1 and type 2 diabetes up to 1 January 2012. Maternal BMI was calculated from height and weight measured at the first antenatal visit. The effect of maternal obesity on offspring outcomes was tested using time-to-event analysis with Cox proportional hazards regression to compare outcomes in offspring of mothers in underweight, overweight or obese categories of BMI, compared with offspring of women with normal BMI.

Results Offspring of obese (BMI $\geq 30 \mathrm{~kg} / \mathrm{m}^{2}$ ) and overweight (BMI $25-29.9 \mathrm{~kg} / \mathrm{m}^{2}$ ) mothers had an increased hazard of type 2 diabetes compared with mothers with normal BMI, after adjustment for gestation when weight was measured, maternal history of diabetes before pregnancy, maternal history of hypertension, age at delivery, parity, socioeconomic status, and sex of the offspring: HR 3.48 (95\% CI 2.33, 5.06) and HR 1.39 (1.06, 1.83), respectively.

Conclusions/interpretation Maternal obesity is associated with increased incidence of type 2 diabetes in the offspring. Evidencebased strategies that reduce obesity among women of reproductive age and that might reduce the incidence of diabetes in their offspring are urgently required.
\end{abstract}

Keywords Diabetes · Obesity · Offspring · Pregnancy

Abbreviations

AMND

Aberdeen Maternity and Neonatal

Databank

Electronic supplementary material The online version of this article (https://doi.org/10.1007/s00125-019-4891-4) contains peer-reviewed but unedited supplementary material, which is available to authorised users.

Rebecca M. Reynolds

R.Reynolds@ed.ac.uk

1 British Heart Foundation Centre for Cardiovascular Science, Queen's Medical Research Institute, University of Edinburgh, 47 Little France Crescent, Edinburgh EH14 6TJ, UK

2 Tommy's Centre for Maternal and Fetal Health, Medical Research Council Centre for Reproductive Health, Queen's Medical Research Institute, University of Edinburgh, Edinburgh, UK
ISD

SCI-Diabetes Scottish Care Information-Diabetes
3 Department of Psychology and Logopedics, Faculty of Medicine, University of Helsinki, Helsinki, Finland

4 Institute of Applied Health Sciences, University of Aberdeen, Aberdeen, UK

5 Usher Institute of Population Health Sciences and Informatics, University of Edinburgh, Edinburgh, UK

6 Institute of Cardiovascular and Medical Sciences, University of Glasgow, Glasgow, UK 


\section{Research in context}

\section{What is already known about this subject?}

- Maternal obesity in pregnancy is associated with cardiovascular disease and mortality rate in the offspring, but the mechanisms are unknown

- It is unclear whether maternal obesity is associated with an increased risk of diabetes in the offspring, as a candidate mechanistic pathway

What is the key question?

- Is maternal obesity associated with increased incidence of type 2 or type 1 diabetes in the offspring?

What are the new findings?

- Women being overweight or obese in pregnancy was associated with over 1.4- and 3.5-fold increased incidence, respectively, of type 2 diabetes in the offspring

How might this impact on clinical practice in the foreseeable future?

- With the rising prevalence of obesity in women of childbearing age, these data highlight the urgent need to establish effective approaches to prevent obesity and diabetes among mothers and their offspring

\section{Introduction}

The short-term pregnancy complications of maternal obesity, including gestational diabetes, pre-eclampsia, need for Caesarean section and delivery of large-for-gestational age infants, are well recognised [1]. There is now increasing awareness that infants born to obese mothers have longerterm health problems, including increased risk of premature cardiovascular disease and premature mortality $[2,3]$. As the prevalence of being overweight or obese among women of childbearing age is increasing worldwide [4], there is an urgent need to determine any long-term adverse effects for the offspring.

One key mechanistic pathway that may explain the link between maternal obesity and offspring cardiovascular disease $[2,3]$ is through increased risk of offspring type 2 diabetes. In support of this, higher maternal BMI was associated with cardiovascular risk factors, including increased fasting insulin (a surrogate marker of insulin resistance), among offspring aged 32 years in the Jerusalem Perinatal Family Follow-up Study [5]. In longer follow-up of older individuals born between 1934 and 1944 in the Helsinki Birth Cohort Study, mothers being overweight was associated with increased risk of offspring diabetes, particularly among female offspring [3]. In this study, however, the diagnosis of diabetes was based on the use of medication and so did not include individuals treated with diet alone and further could not distinguish between diagnoses of type 2 and type 1 diabetes. High maternal BMI has been identified as a risk factor for childhood-onset offspring type 1 diabetes in some studies $[6-8]$ but not in others $[9,10]$.

One small case-control study reported a link between maternal retrospective recall of obesity in pregnancy and offspring type 2 diabetes in adolescence [11]. Another small study, conducted in South India, including follow-up of 204 individuals, found that increased maternal weight measured during pregnancy predicted higher risk of type 2 diabetes in adult offspring [12], although in this study the mean maternal weight was $47 \mathrm{~kg}$. Yet, to our knowledge, no studies have specifically examined whether maternal obesity measured during pregnancy increases the risk of clinician-diagnosed type 2 diabetes in the offspring. Further, although $50 \%$ of type 1 diabetes develops after age 16 years [13], we are not aware of any studies examining whether the previously reported association between maternal obesity and increased risk of offspring childhood-onset type 1 diabetes [6-8] extends to type 1 diabetes diagnosed in adulthood.

We hypothesised that maternal obesity would increase the incidence of subsequent development of both type 2 and type 1 diabetes in the offspring. To test this hypothesis we examined the risk of developing a clinically confirmed diagnosis of type 2 or type 1 diabetes in 118,201 individuals aged 162 years whose mothers' BMI was measured in pregnancy. We used a large pregnancy database, the Aberdeen Maternity and Neonatal Databank (AMND), linked to the Scottish Care Information-Diabetes (SCI-Diabetes) database, a national dataset for people with diagnosed diabetes in Scotland.

\section{Methods}

In this cohort analysis, the exposure was maternal obesity during pregnancy and the outcomes in the offspring were: (1) any diabetes; (2) type 1 diabetes; (3) type 2 diabetes. We created a linked dataset including data from 118,201 mothers 
and their offspring from the AMND databank linked to the SCI-Diabetes database after excluding neonatal deaths and for missing data for offspring sex, gestational age at delivery and year of diabetes diagnosis (Fig. 1). The AMND is a unique databank linking all the obstetric and fertility-related events occurring in women from a defined geographical area with a relatively stable population, between 1950 and the present day [14]. Completeness of the database is checked annually against the number of deliveries recorded in the National Health Service records office, and validity of data entry is verified by numerous consistency checks. All women who delivered a live singleton infant at term ( $\geq 37$ weeks' gestation) between 1950 and 2011, and who had their weight recorded at the first antenatal visit, were identified from the AMND databank. The women were grouped according to their BMI, calculated from height and weight measurements taken at the first antenatal visit, according to WHO criteria: underweight (BMI $<18.5 \mathrm{~kg} / \mathrm{m}^{2}$ ), normal weight (BMI $18.5-24.9 \mathrm{~kg} / \mathrm{m}^{2}$ ), overweight (BMI $25-29.9 \mathrm{~kg} / \mathrm{m}^{2}$ ) and obese (BMI $\geq 30 \mathrm{~kg} /$ $\mathrm{m}^{2}$ ). Relevant details about the index pregnancy were retrieved from the AMND, including maternal age, parity, area-based socioeconomic status, prior self-reported history of diabetes, history of hypertension, gestation at which weight was measured, gestation at delivery, and offspring birthweight, sex and date of birth, from which age at linkage was calculated.

Offspring birth records were linked to a 2011 extract of SCI-Diabetes using the Community Health Index number, a

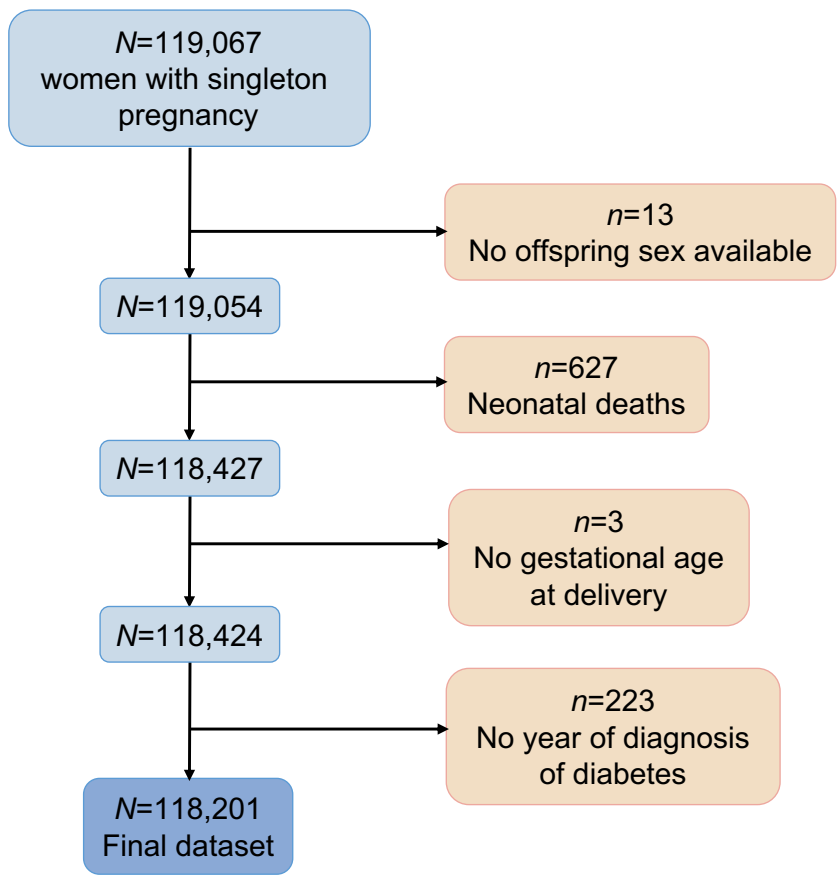

Fig. 1 Flowchart. A linked dataset was created from 118,201 mothers and their offspring from the AMND databank linked to the SCI-Diabetes database, after excluding neonatal deaths and missing data for offspring sex, gestational age at delivery and year of diabetes diagnosis unique individual identifier used on health records in Scotland, or, if this was unavailable, by probabilistic matching on surname, date of birth and postcode (zipcode), to identify individuals with a diagnosis of diabetes.

The SCI-Diabetes database was established at a national level in 2000 and contains demographic and clinical data relevant to diabetes care. It is populated by daily downloads from primary and secondary care databases across Scotland, with increasing completeness since 2004. A validation study among the subset of people with diabetes mentioned on a hospital record in 2007 found that $99 \%$ were included in the diabetes register [15]. In SCI-Diabetes, type of diabetes (type 1 , type 2 , etc.) is recorded by clinicians using a drop-down menu in the electronic health records. To improve the accuracy of this variable for research purposes, an algorithm which combines information from the clinician-recorded diabetes type variable and prescription data is used $[16,17]$. Thus, in the current dataset the date of diabetes diagnoses in the offspring ranged from 1956 to 2011, with diagnoses available from birth for all those born after 2000.

Ethics approval for the creation and analysis of the linked dataset containing no personal identifying information was obtained from the London-Dulwich Research Ethics Committee (reference 16/LO/1410). Approvals were also obtained from the steering committee of the AMND, the Scottish Diabetes Research Network epidemiology subgroup committee and the privacy advisory committee of Information Services Division (ISD) Scotland. The pseudonymised dataset was stored and analysed in the Grampian Data Safe Haven.

Statistical analysis Data were analysed using IBM SPSS Statistics version 24 (IBM, Armonk, NY, USA). Differences in maternal and offspring characteristics according to BMI category were tested using ANOVA for continuous variables and $\chi^{2}$ tests for categorical variables. The effect of maternal obesity on offspring incidence of any diabetes, type 1 diabetes or type 2 diabetes was tested using Cox proportional hazards models, stratifying for year of birth and adjusting for sex of the offspring, comparing outcomes in the offspring of mothers whose pregnancy BMI was underweight, overweight or obese, with those of women with normal BMI. Analyses were adjusted for potential confounding factors, including maternal history of diabetes before pregnancy, maternal history of hypertension, maternal age at delivery, gestation when weight was measured, deprivation category and parity. For the two variables with missing data, on deprivation category and gestation when weight was measured, the missing data were coded to their own categories. In sensitivity analyses we first repeated the analyses after exclusion of women with a history of diabetes prior to pregnancy, to study associations between maternal obesity and offspring outcomes in women without known diabetes. We then repeated the analyses limiting them to those born between 1950 and 1976, i.e. with similar 
duration of follow-up to those in whom we observed a link between mothers being overweight or obese and offspring cardiovascular events and mortality rates [2]. In addition, we repeated the analyses after excluding women with diabetes prior to pregnancy and with diabetes diagnosed during or after pregnancy. Finally, we examined interactions by sex and repeated the analyses separately for male and female offspring. These interaction models were adjusted for the main effects of sex and maternal BMI.

\section{Results}

Table 1 shows the characteristics of the 118,201 mothers and their offspring. Among the mothers, $24.8 \%$ were overweight and $9.5 \%$ were obese. The proportion of mothers who were obese increased fivefold between 1950 and 1959 and 2000 and 2011 from $3.1 \%(n=468)$ to $15.7 \%(n=4490)$ $(p<0.0001)$. Obese women were older and had higher parity and lower deprivation category. The gestation at which weight was first measured was significantly later in overweight and obese women. Among the offspring, 732 (0.6\%) had a record of diagnosed diabetes. Among offspring with diabetes, offspring of obese mothers had the highest BMI. More male offspring were diagnosed with diabetes, compared with female offspring, across all maternal BMI categories.

\section{Maternal obesity and any diabetes in the offspring}

Table 2 shows the HRs and 95\% CIs for any diabetes in offspring according to maternal BMI category. There was a significant increase in HR of any diabetes in the offspring of mothers who were overweight or obese. The associations were independent of all assessed covariates: after adjustment for gestation when weight was measured, maternal age at delivery, parity, deprivation category, maternal history of diabetes before pregnancy and sex of the offspring, mother being overweight or obese was still associated with over 1.3- and 1.8-fold increased HR, respectively, of any diabetes in the offspring.

Maternal obesity and type 1 diabetes in offspring Overall, $410(0.3 \%)$ of the offspring had a diagnosis of type 1 diabetes. Age at diagnosis of type 1 diabetes ranged from 1 to 52 years. Table 2 shows the HRs and 95\% CIs for type 1 diabetes in the offspring according to maternal BMI category. In unadjusted analyses and analyses adjusted for potential confounding factors, there was no association between mothers being overweight or obese and HR of type 1 diabetes in the offspring.

Maternal obesity and type 2 diabetes in offspring Among the offspring, $319(0.3 \%)$ had a diagnosis of type 2 diabetes. Age at diagnosis of type 2 diabetes ranged from 10 to 61 years.
Table 2 and Fig. 2 show the HRs and 95\% CIs for type 2 diabetes in the offspring according to maternal BMI category. There was a significant increase in the HR of type 2 diabetes in the offspring of mothers who were overweight or obese. The associations were independent of all assessed covariates: after adjustment for covariates, mothers being overweight or obese was still associated with over 1.4- and 3.5-fold increased HRs, respectively, of type 2 diabetes in the offspring.

Sensitivity analyses Associations between maternal obesity and any diabetes and type 2 diabetes in the offspring remained significant when the 385 women with a history of diabetes prior to pregnancy were excluded from the analyses (electronic supplementary material [ESM] Table 1). These associations also remained significant after excluding all women with diabetes before, during or after pregnancy (ESM Table 1). When restricting the analyses to offspring born between 1950 and 1976, maternal obesity was associated with a 2.3-fold increased HR of offspring type 1 diabetes, as well as a 3.7-fold increased HR of offspring type 2 diabetes and a 3.3-fold HR of any diabetes (ESM Table 2). In individuals born after 1977, maternal obesity was associated with a 2.6-fold increased HR of type 2 diabetes.

Sex differences in outcomes While the overall interactions between maternal BMI and sex of the offspring in predicting offspring HR of any diabetes and type 2 diabetes were marginal ( $p=0.084$ and $p=0.076)$, there were significant interactions specifically for maternal overweight vs normal weight and sex of the offspring in predicting any diabetes $(p=0.029$ for interaction) and type 2 diabetes ( $p=0.015$ for interaction) in the offspring. Offspring any diabetes and type 2 diabetes were more strongly associated with mothers being overweight in female than in male offspring. The HRs and 95\% CIs for any diabetes and type 2 diabetes in offspring born to overweight mothers were $1.50(1.12,2.01)$ and $2.26(1.37,3.72)$ for female offspring and $1.11(0.89,1.37)$ and $1.11(0.81$, $1.53)$ for male offspring, respectively. There were no interactions by sex on offspring risk of type 1 diabetes $(p \geq 0.407)$.

\section{Discussion}

In this large cohort study including over 60 years of pregnancy data linked to the national diabetes dataset we found that, for pregnant women, being overweight or obese was associated with an increased incidence of type 2 diabetes in the offspring. This association with type 2 diabetes may lie on the causal pathway to explain the link between being overweight or obese during pregnancy and offspring cardiovascular disease and mortality rates $[2,3]$, and it highlights a potential target for intervention. 
Table 1 Characteristics of 118,201 mothers and their offspring according to maternal BMI

\begin{tabular}{|c|c|c|c|c|c|}
\hline Characteristic $^{\mathrm{a}}$ & $\begin{array}{l}\text { Underweight } \\
n=5283\end{array}$ & $\begin{array}{l}\text { Normal weight } \\
n=72,329\end{array}$ & $\begin{array}{l}\text { Overweight } \\
n=29,325\end{array}$ & $\begin{array}{l}\text { Obese } \\
n=11,264\end{array}$ & $\begin{array}{l}p \\
\text { value }\end{array}$ \\
\hline \multicolumn{6}{|l|}{ Mothers } \\
\hline Age, years & $24.8(5.2)$ & $26.1(5.5)$ & $27.1(5.6)$ & $27.7(5.6)$ & $<0.001$ \\
\hline Primiparous, $n(\%)$ & $4146(78.5)$ & $54,611(75.5)$ & $20,807(71.0)$ & $7963(70.7)$ & $<0.001$ \\
\hline \multicolumn{6}{|l|}{ Deprivation category $^{\mathrm{b}}$} \\
\hline $1-2, n(\%)$ & $1708(32.3)$ & $22,357(30.9)$ & $7057(24.0)$ & $1690(15.0)$ & \multirow[t]{3}{*}{$<0.001$} \\
\hline $3-4, n(\%)$ & $1481(28.0)$ & $23,663(32.7)$ & $10,783(36.8)$ & $4427(39.3)$ & \\
\hline $5-6, n(\%)$ & $1391(26.3)$ & $15,477(21.4)$ & $7000(23.9)$ & $1784(15.8)$ & \\
\hline Gestational weight measured, weeks & $12.23(4.2)$ & $14.22(6.7)$ & $16.21(8.6)$ & $15.64(9.2)$ & $<0.001$ \\
\hline History of diabetes before pregnancy, $n(\%)$ & $3(0.06)$ & $148(0.2)$ & $157(0.5)$ & $77(0.7)$ & $<0.001$ \\
\hline History of hypertension, $n(\%)$ & $117(2.2)$ & $1904(2.6)$ & $924(3.2)$ & $383(3.4)$ & $<0.001$ \\
\hline Any diabetes, $n(\%)$ & $152(2.9)$ & $2033(2.8)$ & $1284(4.4)$ & $860(7.6)$ & $<0.001$ \\
\hline \multicolumn{6}{|l|}{ Offspring } \\
\hline \multicolumn{6}{|l|}{ Any diabetes } \\
\hline Total, $n(\%)$ & $31(0.59)$ & $437(0.60)$ & $187(0.64)$ & $77(0.68)$ & 0.735 \\
\hline Age at diagnosis, years & $32.1(21.1)$ & $28.5(19.1)$ & $27.0(18.3)$ & $26.2(17.2)$ & 0.393 \\
\hline BMI, $\mathrm{kg} / \mathrm{m}^{2}$ & $27.1(6.8)$ & $28.4(7.2)$ & $29.7(7.5)$ & $32.2(9.2)$ & $<0.001$ \\
\hline Male, $n(\%)$ & $24(77.4)$ & $309(70.7)$ & $118(63.1)$ & $55(71.4)$ & 0.181 \\
\hline \multicolumn{6}{|l|}{ Type 1 diabetes } \\
\hline Total, $n(\%)$ & $14(0.27)$ & $242(0.33)$ & $112(0.38)$ & $42(0.37)$ & 0.458 \\
\hline Age at diagnosis, years & $11.1(8.5)$ & $14.3(10.4)$ & $14.5(9.6)$ & $13.3(8.6)$ & 0.621 \\
\hline BMI, $\mathrm{kg} / \mathrm{m}^{2}$ & $22.2(3.8)$ & $24.7(5.3)$ & $25.6(4.5)$ & $26.9(5.8)$ & 0.015 \\
\hline Male, $n(\%)$ & $10(71.4)$ & $155(64.0)$ & $68(60.7)$ & $27(64.3)$ & 0.851 \\
\hline \multicolumn{6}{|l|}{ Type 2 diabetes } \\
\hline Total, $n(\%)$ & $17(0.32)$ & $193(0.27)$ & $74(0.25)$ & $35(0.31)$ & 0.664 \\
\hline Age at diagnosis, years & $49.3(8.7)$ & $46.2(11.0)$ & $45.4(10.8)$ & $41.7(11.0)$ & 0.068 \\
\hline BMI, $\mathrm{kg} / \mathrm{m}^{2}$ & $30.9(6.2)$ & $32.7(6.7)$ & $34.9(7.5)$ & $37.6(8.9)$ & 0.001 \\
\hline Male, $n(\%)$ & $14(82.4)$ & $153(79.3)$ & $49(66.2)$ & $28(80.0)$ & 0.125 \\
\hline
\end{tabular}

Data are mean (SD), except where indicated as $n(\%)$

${ }^{\text {a }}$ Complete data available for all variables other than deprivation category and gestation weight measured where data were available for 100,397 and 112,340 women, respectively

${ }^{\mathrm{b}}$ Deprivation category according to area socioeconomic status: $1-2$, most deprived; 3-4, middle; 5-6, least deprived

Our finding of a link between being overweight or obese and type 2 diabetes in the offspring is in accord with previous observations of associations of pregnant women being overweight and higher maternal weight with increased incidence of type 2 diabetes in the offspring [11, $12]$, and of maternal obesity with markers in the offspring of increased insulin resistance in childhood [18] and young adulthood $[5,11,19]$; it is also consistent with the findings of increased diabetes risk in offspring born to overweight mothers in the Helsinki Birth Cohort Study [3]. Although the latter study could not distinguish between types of diabetes, the association between being overweight and risk of diabetes was stronger in women than in men, as observed in our dataset. The reasons for a sex difference in the findings are unknown but are possibly related to the smaller pancreatic volume [20], and hence beta cell reserve, in women compared with men, potentially programmed in utero [21], and the findings are consistent with those of other studies suggesting women may be more vulnerable to adverse metabolic programming influences [22, 23]. Evidence suggests increasing incidence of type 1 diabetes in children born between 1989 and 2003 [24], a time when rates of maternal obesity were dramatically rising, as also observed in our dataset. Associations between maternal obesity and childhoodonset type 1 diabetes have been reported in some studies [6-8] but not others [9, 10]. Notably, two studies from Sweden observed an increased incidence of childhood 
Table 2 Offspring diabetes according to maternal BMI

\begin{tabular}{|c|c|c|c|c|c|c|}
\hline Offspring diabetes & HR & $95 \% \mathrm{CI}$ & $p$ value & Adjusted HR & $95 \% \mathrm{CI}$ & $p$ value \\
\hline \multicolumn{7}{|l|}{ Any diabetes } \\
\hline Underweight & 0.96 & $0.67,1.38$ & 0.829 & 0.94 & $0.65,1.36$ & 0.739 \\
\hline Normal weight & 1 & & & 1 & & \\
\hline Overweight & 1.23 & $1.03,1.46$ & 0.020 & 1.26 & $1.06,1.51$ & 0.009 \\
\hline Obese & 1.78 & $1.38,2.26$ & $<0.001$ & 1.83 & $1.42,2.35$ & $<0.001$ \\
\hline \multicolumn{7}{|l|}{ Type 1 diabetes } \\
\hline Underweight & 0.88 & $0.51,1.51$ & 0.639 & 0.88 & $0.51,1.50$ & 0.628 \\
\hline Normal weight & 1 & & & 1 & & \\
\hline Overweight & 1.14 & $0.91,1.42$ & 0.27 & 1.16 & $0.92,1.46$ & 0.204 \\
\hline Obese & 1.23 & $0.88,1.70$ & 0.23 & 1.25 & $0.89,1.75$ & 0.192 \\
\hline \multicolumn{7}{|l|}{ Type 2 diabetes } \\
\hline Underweight & 1.07 & $0.65,1.76$ & 0.78 & 1.05 & $0.64,1.74$ & 0.835 \\
\hline Normal weight & 1 & & & 1 & & \\
\hline Overweight & 1.34 & $1.03,1.75$ & 0.032 & 1.39 & $1.06,1.83$ & 0.018 \\
\hline Obese & 3.33 & $2.31,4.78$ & $<0.001$ & 3.48 & $2.33,5.06$ & $<0.001$ \\
\hline
\end{tabular}

Adjusted HR, analyses were adjusted for maternal history of diabetes before pregnancy, maternal history of hypertension, maternal age at delivery, gestation when weight was measured, deprivation category, parity and sex of offspring type 1 diabetes in obese mothers without diabetes $[6,8]$. We were unable to replicate this finding in our dataset. Reasons for the differences between the Swedish and Aberdeen cohorts are unknown and we only found an association of maternal obesity with offspring type 1 diabetes when we restricted the cohort to individuals who had at least 35 years of follow-up.

The underlying mechanisms linking mothers being overweight/obese to type 2 diabetes in the offspring are not known. The fetal overnutrition hypothesis suggests that the adverse in utero environment associated with maternal obesity, including high circulating levels of glucose, insulin and NEFA, programmes adverse offspring outcomes [25]. In addition, obesity in pregnancy is associated with complex neuroendocrine,

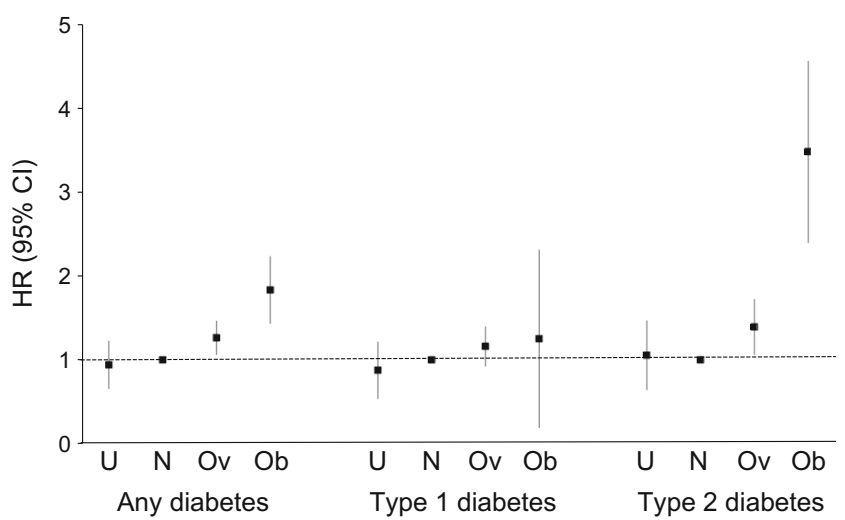

Fig. 2 Offspring diabetes according to maternal BMI. U, underweight; $\mathrm{N}$, normal weight; Ov, overweight; Ob, obese metabolic and immune/inflammatory changes which likely impact on fetal hormonal exposure and nutrient supply [26, 27]. It is also thought that epigenetic factors in the intrauterine environment of obese mothers may initiate beta cell stress, metabolic dysregulation and earlier onset of type 2 diabetes and cardiometabolic risk [28]. Further, although we adjusted for both prenatal and postnatal environmental influences in our statistical analyses, it is extremely likely that there will be residual confounding by environmental factors that we were unable to measure. The Hyperglycaemia and Adverse Pregnancy Outcome (HAPO) study showed an association between increased maternal BMI and fetal hyperinsulinaemia, independently of maternal glycaemia [29], supporting an intrauterine influence of maternal obesity on glucose metabolism in the offspring. Others reported that rapid growth in the postnatal environment from birth onwards, rather than during fetal life, was associated with higher insulin levels in childhood [30]. Further studies are needed to understand the contribution of pre- and postnatal mechanisms linking maternal obesity with type 2 diabetes in the offspring.

The strengths of our study include the large sample size and the detailed quality of the antenatal records, including maternal history of type 1 diabetes. Our findings remained similar if we adjusted for maternal diabetes prior to pregnancy in the analyses or excluded women with a history of diabetes both prior to and after pregnancy from the analyses. In addition, we used national population-based health data for diagnosed diabetes (SCI-Diabetes), which allowed us to examine outcomes of both type 2 and type 1 diabetes in the same dataset. A further major strength of our study is that we included outcome data for offspring from birth to age 62 years, which 
allowed us to explore outcomes across the lifespan. Scottish Diabetes Survey data indicate that type 2 diabetes prevalence increases with age to about $11 \%$ for 55 - to 59 -year-olds (i.e. the oldest age group in this study) [13]. To our knowledge, there are no other databases worldwide that would allow such linkage between maternal obesity records in pregnancy and confirmed diagnosis of diabetes in the offspring. The main limitation is that as only maternal history of type 1 diabetes is recorded in the AMND, we were not able to use 'gestational diabetes' or 'type 2 diabetes' as covariates to determine whether there was a difference in offspring outcomes of mothers who were obese but did not have diabetes during pregnancy or who were both obese and had gestational or type 2 diabetes. However, we believe the number of women with pre-existing type 2 diabetes in this dataset would be negligible, particularly for mothers who were pregnant in the 1950s and 1960s. In addition, the definition of gestational diabetes has changed over the study period and more detailed measurements of glucose levels taken during pregnancy would be required to generate a comparable definition. As hypertension is part of the metabolic syndrome, we included history of hypertension in our models as a proxy for insulin resistance. Data on maternal gestational weight gain were not available, although a recent study found no evidence that gestational weight gain influenced risk of childhood type 1 diabetes [8]. A further limitation of our study is that we did not have data for all offspring on BMI or lifestyle factors (e.g. diet, exercise) that are known to increase the risk of type 2 diabetes. Indeed, it is plausible that our observations were due to increased BMI in the child, either through prenatal programming of BMI or through shared lifestyle of mother and child. In either case, our observations are important, as pregnancy represents a potential time to intervene with health advice for the family.

In conclusion, we showed that, in pregnant women, being overweight or obese was associated with increased type 2 diabetes incidence in the offspring, independently of perinatal and sociodemographic covariates and maternal history of diabetes. With the rising prevalence of being overweight/obese in women of childbearing age (for example, recent data indicated that over $60 \%$ of women in the USA were overweight or obese at the time of conception [4]), our findings have profound public health implications. There is an urgent need to establish effective approaches to prevention of obesity and diabetes among mothers and their offspring.

Acknowledgements We thank the AMND data management team (University of Aberdeen) and S. Nowell (ISD Scotland) for their help with data extraction and linkage. Some of the data were presented as an abstract at the 10th World Congress on Developmental Origins of Health and Disease in 2017. However, the work is not under consideration for publication with any other journal.

Data availability Data are stored in the Grampian Data Safe Haven and are available on request.
Funding This work was supported by Tommy's Centre for Maternal and Fetal Health and the Medical Research Council (MR/N022556/1). We acknowledge the support of the British Heart Foundation.

The study sponsors had no role in the study design, data collection and analysis, decision to publish or preparation of the manuscript.

Duality of interest The authors declare that there is no duality of interest associated with this manuscript.

Contribution statement RMR designed the study, analysed the data and wrote the manuscript. ML-P conducted the statistical analysis and wrote the manuscript. SoB, SHW, RSL, KR, JEN and SiB designed the study and commented on the manuscript. All authors approved the final version of the manuscript. RMR is guarantor of the study and takes full responsibility for the work as a whole, including the study design, access to data and the decision to submit and publish the manuscript.

Open Access This article is distributed under the terms of the Creative Commons Attribution 4.0 International License (http:// creativecommons.org/licenses/by/4.0/), which permits unrestricted use, distribution, and reproduction in any medium, provided you give appropriate credit to the original author(s) and the source, provide a link to the Creative Commons license, and indicate if changes were made.

\section{References}

1. Norman JE, Reynolds RM (2011) The consequences of obesity and excess weight gain in pregnancy. Proc Nutr Soc 70(04):450-456. https://doi.org/10.1017/S0029665111003077

2. Reynolds RM, Allan KM, Raja EA et al (2013) Maternal obesity during pregnancy and premature mortality from cardiovascular event in adult offspring: follow-up of 1323275 person years. Br Med J 347:f4539. https://doi.org/10.1136/bmj.f4539

3. Eriksson JG, Sandboge S, Salonen MK, Kajantie E, Osmond C (2014) Long term consequences of maternal overweight in pregnancy on offspring later health: findings from the Helsinki Birth Cohort Study. Ann Med 46(6):434-438. https://doi.org/10.3109/ 07853890.2014 .919728

4. Flegal KM, Carroll MD, Kit BK, Ogden CL (2012) Prevalence of obesity and trends in the distribution of body mass index among US adults, 1999-2010. JAMA 307(5):491-497. https://doi.org/10. 1001/jama.2012.39

5. Hochnher H, Friedlander Y, Calderon-Margalit R et al (2012) Associations of maternal prepregnancy body mass index and gestational weight gain with adult offspring cardiometabolic risk factors: the Jerusalem Perinatal Family Follow-up Study. Circulation 125(11): 1381-1389. https://doi.org/10.1161/CIRCULATIONAHA.111. 070060

6. Hussen HI, Persson M, Moradi T (2015) Maternal overweight and obesity are associated with increased risk of type 1 diabetes in offspring of parents without diabetes regardless of ethnicity. Diabetologia 58(7):1464-1473. https://doi.org/10.1007/s00125015-3580-1

7. D'Angeli MA, Merzon E, Valbuena LF, Tirschwell D, Paris CA, Mueller BA (2010) Environmental factors associated with childhood-onset type 1 diabetes mellitus: an exploration of the hygiene and overload hypotheses. Arch Pediatr Adolesc Med 164(8): 732-738. https://doi.org/10.1001/archpediatrics.2010.115

8. Lindell N, Carlsson A, Josefsson A, Samuelsson U (2018) Maternal obesity as a risk factor for early childhood type 1 diabetes: a nationwide, prospective, population-based case-control study. 
Diabetologia 61(1):130-137. https://doi.org/10.1007/s00125-0174481-2

9. Arkkola T, Kautjainen S, Takkinen HM et al (2011) Relationship of maternal weight status and weight gain rate during pregnancy to the development of advanced beta cell autoimmunity in the offspring: a prospective birth cohort study. Pediatr Diabetes 12(5):478-484. https://doi.org/10.1111/j.1399-5448.2010.00703.x

10. Robertson L, Harrild K (2010) Maternal and neonatal risk factors for childhood type 1 diabetes: a matched case-control study. BMC Public Health 10(1):281. https://doi.org/10.1186/1471-2458-10281

11. Dabelea D, Mayer-Davis EJ, Lamichhane AP et al (2008) Association of intrauterine exposure to maternal diabetes and obesity with type 2 diabetes in youth: the SEARCH Case-Control Study. Diabetes Care 31(7):1422-1426. https://doi.org/10.2337/ $\mathrm{dc} 07-2417$

12. Fall CHD, Stein CE, Kumaran K et al (1998) Size at birth, maternal weight, and type 2 diabetes in South India. Diabet Med 15(3):220 227. https://doi.org/10.1002/(SICI)1096-9136(199803)15:3<220:: AID-DIA544>3.0.CO;2-O

13. Scottish Diabetes Data Group (2017) Scottish Diabetes Survey. Available from www.diabetesinscotland.org.uk/Publications/SDS 2017.pdf accessed 20 September 2018

14. Ayorinde AA, Wilde K, Lemon J, Campbell D, Bhattacharya S (2016) Data resource profile: the Aberdeen Maternity and Neonatal Databank (AMND). Int J Epidemiol 45(2):389-394. https://doi.org/10.1093/ije/dyv356

15. Anwar H, Fischbacher CM, Leese GP et al (2011) Assessment of the under-reporting of diabetes in hospital admission data: a study from the Scottish Diabetes Research Network Epidemiology Group. Diabet Med 28(12):1514-1519. https://doi.org/10.1111/j. 1464-5491.2011.03432.x

16. Read SH, Kerssens JJ, McAllister DA et al (2016) Trends in type 2 diabetes incidence and mortality in Scotland between 2004 and 2013. Diabetologia 59(10):2106-2113. https://doi.org/10.1007/ s00125-016-4054-9

17. Livingstone SJ, Looker HC, Hothersall EJ et al (2012) Risk of cardiovascular disease and total mortality in adults with type 1 diabetes: Scottish registry linkage study. PLoS Med 9(10): e1001321. https://doi.org/10.1371/journal.pmed.1001321

18. Boney CM, Verma A, Tucker R, Vohr BR (2005) Metabolic syndrome in childhood: association with birthweight, maternal obesity, and gestational diabetes mellitus. Pediatrics 115(3):e290-e296. https://doi.org/10.1542/peds.2004-1808

19. Mingrone G, Manco M, Mora ME et al (2008) Influence of maternal obesity on insulin sensitivity and secretion in offspring. Diabetes Care 31(9):1872-1876. https://doi.org/10.2337/dc080432

20. Burute N, Nisenbaum R, Jenkins DJ et al (2014) Pancreas volume measurement in patients with type 2 diabetes using magnetic resonance imaging-based planimetry. Pancreatology 14(4):268 274. https://doi.org/10.1016/j.pan.2014.04.031

21. Calzada L, Morales A, Sosa-Larios TC et al (2016) Maternal protein restriction during gestation impairs female offspring pancreas development in the rat. Nutr Res 36(8):855-862. https://doi.org/10. 1016/j.nutres.2016.03.007

22. Carpenter T, Grecian SM, Reynolds RM (2017) Sex differences in early-life programming of the hypothalamic-pituitary-adrenal axis in humans suggest increased vulnerability in females: a systematic review. J Dev Orig Health Dis 8(2):244-255. https://doi.org/10. 1017/S204017441600074X

23. Aldhous M, Reynolds RM, Campbell A et al (2015) Sexdifferences in the metabolic health of offspring of parents with diabetes: a record-linkage study. PLoS One 10(8):e0134883. https://doi.org/10.1371/journal.pone.0134883

24. Patterson CC, Dahlquist GG, Gyürüs E, Green A, Soltész G, the EURODIAB Study Group (2009) Incidence trends for childhood type 1 diabetes in Europe during 1989-2003 and predicted new cases 2005-20: a multicentre prospective registration study. Lancet 373(9680):2027-2033. https://doi.org/10.1016/S01406736(09)60568-7

25. Taylor PD, Poston L (2007) Developmental programming of obesity in mammals. Exp Physiol 92(2):287-298. https://doi.org/10. 1113/expphysiol.2005.032854

26. Stirrat LI, O'Reilly JR, Barr SM et al (2015) Decreased maternal hypothalamic-pituitary-adrenal axis activity in very severely obese pregnancy: associations with birthweight and gestation at delivery. Psychoneuroendocrinol 63:135-143

27. Forbes S, Barr SM, Reynolds RM et al (2015) Convergence in insulin resistance between very severely obese and lean women at the end of pregnancy. Diabetologia 58(11):2615-2626. https://doi. org/10.1007/s00125-015-3708-3

28. Godfrey KM, Reynolds RM, Prescott SL et al (2017) Influence of maternal obesity on the long-term health of offspring. Lancet Diabetes Endocrinol 5(1):53-64. https://doi.org/10.1016/S22138587(16)30107-3

29. HAPO Study Cooperative Research Group (2010) Hyperglycaemia and Adverse Pregnancy Outcome (HAPO) study: associations with maternal body mass index. BJOG 117(5):575-584. https://doi.org/ 10.1111/j.1471-0528.2009.02486.x

30. Voerman E, Jaddoe VW, Franco OH, Steergers EA, Gaillard R (2017) Critical periods and growth patterns from fetal life onwards associated with childhood insulin levels. Diabetologia 60(1):81-88. https://doi.org/10.1007/s00125-016-4135-9

Publisher's note Springer Nature remains neutral with regard to jurisdictional claims in published maps and institutional affiliations. 